\title{
Cavernous hemangioma arising in an intramural leiomyoma
}

\author{
Zeliha Esin Celik ${ }^{*}$, Senem Bastoklu' ${ }^{2}$, Tolgay Tuyan Ilhan ${ }^{3}$ and Cetin Celik ${ }^{3}$ \\ ${ }^{1}$ Faculty of Medicine, Department of Pathology, Selcuk University, Turkey \\ ${ }^{2}$ Resident, Aksehir State Hospital, Department of Pathology, Turkey \\ ${ }^{3}$ Faculty of Medicine, Department of Obstetrics and Gyneacology, Selcuk University, Turkey
}

\begin{abstract}
Hemangiomas, or benign tumors of blood vessels, rarely arise in the uterus; however, they can lead to many clinical complications such as abdominal pain and excessive vaginal bleeding. They can occur at any age but they primarily affect pregnant women. Herein, we report a 38- year-old non-pregnant woman who presented with vaginal bleeding and underwent hysterectomy after detection of a $5.5-\mathrm{cm}$ intramural mass diagnosed as a "cavernous hemangioma arising in intramural leiomyoma" after pathologic examination. To the best of our knowledge, this is the first case of uterine hemangioma and intramural leiomyoma coexistence. The possibility of such ocurrence must be kept in mind in differential diagnosis of patients with abnormal vaginal bleeding.
\end{abstract}

\section{Introduction}

A cavernous hemangioma located in the uterus is an extremely rare condition and very few incidents have been reported in the literature. Many of such uterine hemangiomas have been detected in pregnant women with complications such as uncontrolled bleeding or pain $[1,2]$.

Some extreme localizations, such as the cervical region [3] or adenomyotic foci in the uterus [1], have been reported for uterine hemangiomas. In this case, we describe a cavernous hemangioma arising in an uterine intramural leiomyoma detected in a non-pregnant woman. To our knowledge, this case is unique in the current literature in the presentation of this combination.

\section{Case report}

A 38-year-old woman was admitted to the hospital with abnormal vaginal bleeding. The patient reported three prior vaginal deliveries, with her last pregnancy occurring two years prior to her hospitalization. She further reported suffering from menorrhagia and dysmenorrhea since her last delivery. On physical examination of the patient, a cervical polyp lying to vagina from cervical os was detected and the uterus was found to be larger than normal. Ultrasonography (USG) revealed a $5.5 \times 5.0 \mathrm{~cm}$ mass with cystic components originating from the posterior wall of the uterus and compressing the endometrial cavity. MRI findings were consistent with USG results and revealed the same size intramural leiomyoma with cystic components (Figure 1).

The patient underwent total abdominal hysterectomy due to excessive vaginal bleeding and myoma uteri. Upon gross examination of the uterus, a $2.0 \times 1.5 \mathrm{~cm}$ polyp in the endocervical region and a $5.5 \times 5.0 \mathrm{~cm}$ gray-tan colored intramural nodule with smooth borders in the uterine corpus was detected. There was a $1.4 \times 1.1 \mathrm{~cm}$ area containing blood-filled cystic spaces on the cut surface of the nodule. The nodule consisted of intercrossing smooth muscle bundles with no cellular atypia, mitoses, or necrosis, as determined microscopically. The cystic spaces at the center of the nodule were lined with a single layer of flattened cells and had a back-to-back appearance of thinwalled cavernous structures (Figures 2,3). Immunohistochemical CD31 staining was positive at the lining cells, favoring their endothelial nature (Figure 4). In light of these findings, pathological diagnosis was "cavernous hemangioma arising in an intramural leiomyoma".

The patient was discharged uneventfully and was symptom free at her six-month follow-up.

\section{Discussion}

Hemangiomas are benign tumors originating from endothelial cells of blood vessels [4]. They appear in two forms: capillary and cavernous. They can arise from many organs, such as liver, lung and skin; however, location in the uterus is extremely rare. While rare, both capillary and cavernous hemangiomas may occur in the uterus. Capillary hemangiomas tend to be restricted to the endometrium, while the cavernous form involves the uterus in a diffuse manner $[4,5]$. A localized hemangioma can present as an endometrial polyp or be restricted to the myometrium. It can rarely present as a cavernous hemangiomatous polyp [6].

Uterine hemangioma was first described in 1897 as an incidental finding from an autopsy of a young woman who died 24 hours after delivering twins following severe anemia and dyspnea. Due to the extremely small number of case reports (fewer than 60 cases reported to date), the exact incidence of uterine hamngiomas is still unclear

Correspondence to: Zeliha Esin Celik, Faculty of Medicine, Department of Pathology, Selcuk University, Alaaddin Keykubat Kampus, 42000, Selcuklu, Konya, Turkey, Tel: 0090332 2244813, E-mail: dresincelik@hotmail.com

key words: hemangioma, cavernous, uterus, leiomyoma, vaginal bleeding

Received: February 06, 2017; Accepted: February 26, 2017; Published: February 28,2017 
$[7,8]$. In the present report, a uterine hemangioma arising in myoma uteri is presented as a rare condition.

The clinical symptoms range from none (asymptomatic) to abdominal pain, excessive vaginal bleeding, anemia, infertility, and maternal and pregnancy-associated complications such as postpartum hemorrhage, disseminated intravascular coagulation (DIC), thrombosis with organization, and calcification $[4,5,7,8]$. Many of the reported cases are pregnant woman presenting with a complication of uterine hemangioma [1]. In their short review, Chou et al. [8] reported five Taiwanese women with diffuse uterine hemangioma located in both the uterus and cervix, most of whom were preoperatively diagnosed as adenomyosis. Although most of the uterine hemangiomas were detected in pregnant women, some isolated cases of non- pregnant women with uterine hemangiomas have been described $[1,5,6,9]$. Saeed-Vafa et al. [1] reported a 27-year-old non-pregnant woman with localized cavernous hemangioma in adenomyotic foci in the uterus. Malhotra et al. [9] reported a 14-year-old girl with severe anemia due to bleeding of a uterine cavernous hemangioma. Lee et al. [5] presented a uterine cavernous hemangioma in an 81-year-old female presenting with recurrent menorrhagia and abdominal pain and mass. Sharma et al. [6] reported a 40-year-old woman with perimenopausal bleeding due to uterine hemangioma.

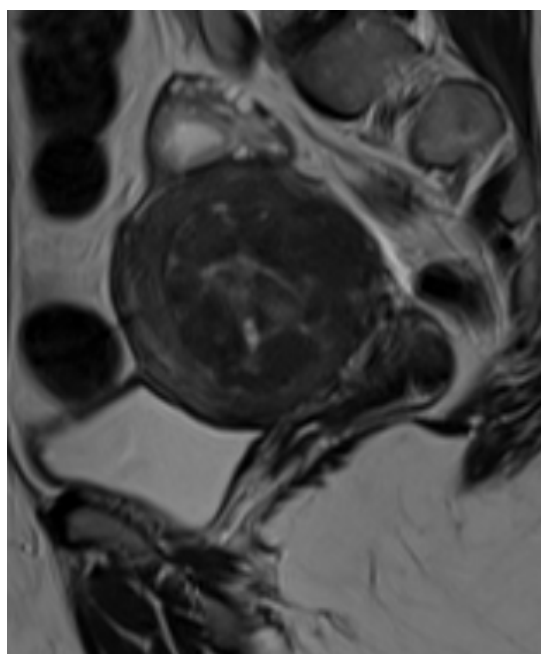

Figure 1. On MRI, a 5,5x5 cms mass with cystic components was seen to be originated from posterior wall of the uterus and compressed the endometrial cavity.

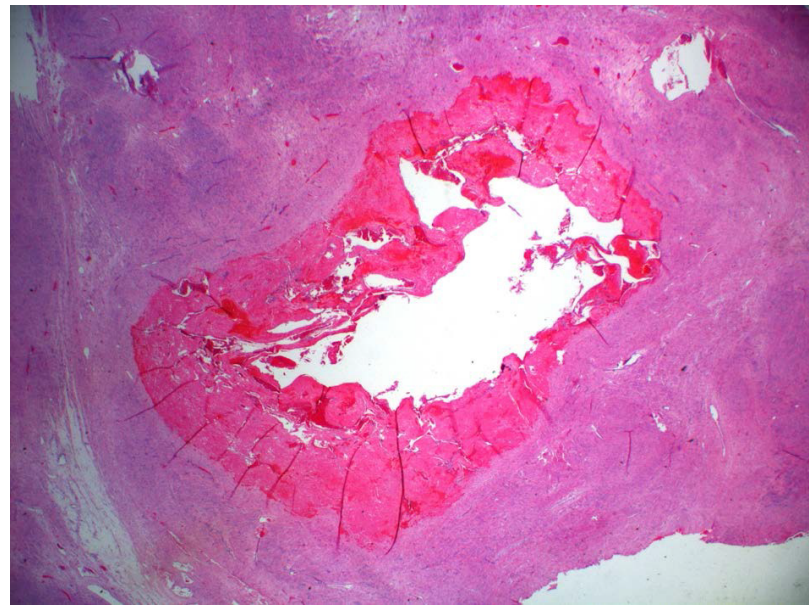

Figure 2. The nodule consisted of intercrossing smooth muscle bundles with a central cystic component. (HEX25).

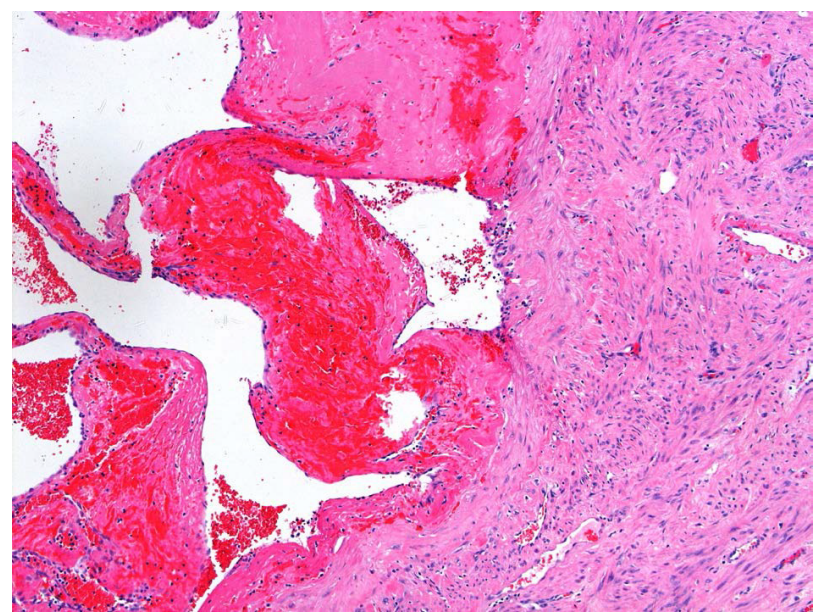

Figure 3. The cystic spaces at the center of the nodule lined with a single layer of flattened cells and had a back to back appearance of thin walled cavernous structures. (HEX100).

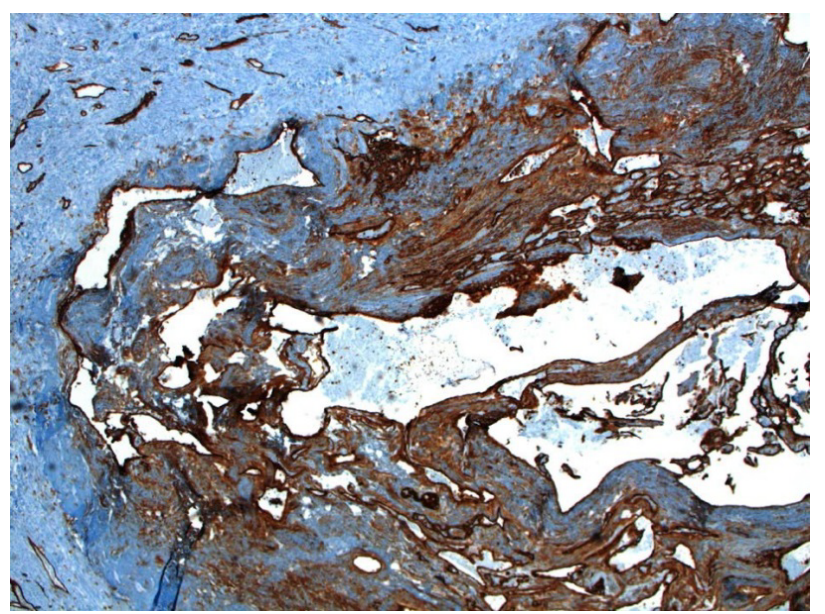

Figure 4. Positive immunohistochemical CD31 staining of the lining cells (X40)

Uterine hemangioma cells possibly originate from embryonic pluripotent mesodermal cells within the uterus $[8,10]$. Uterine hemangiomas may present in either congenital or acquired forms. In the literature, many of the presented cases have been reported as acquired hemangiomas [7,8]. In the pathogenesis of uterine hemangiomas, physical changes including hypoxia, endometrial curettage, previous pelvic surgery, tissue injury, and increased blood volume during pregnancy, as well as some conditions leading to hormone alterations, especially high estrogen levels such as menarche, pregnancy, endometrial cancer, and trophoblastic diseases have been proposed $[4,8]$. Induction of angiogenesis of hemangioma by estrogen has been suggested in some studies, although this hypothesis has not been supported via immunohistochemical methods using estrogen receptors in the study by Chou et al. [8] However, the common existence of uterine hemangioma in pregnant women may be evidence of the influence of estrogen in the development of uterine hemangioma. Any conditions leading to increased estrogen levels may play a possible role in the development of uterine hemangioma in nulliparous women. Additionally, presentation of uterine hemangioma in non- pregnant women such as in our case can be explained by the gradual increase in size of a preexisting lesion following pregnancy.

Differential diagnosis of uterine hemangioma includes benign lesions such as arteriovenous malformation (AVM), lymphangioma, vascular leiomyoma, and hybrid tumor (angiomyofibroma). 
AVMs are thick- and thin-walled arteries and veins in close association with one another forming a fistula. Within the lesion, many thin-walled capillaries interlacing muscular vessels are encountered [7]. In our case, the back-to-back appearance of thin-walled cavernous structures in a leiomyomatous background and the absence of capillarysized vessels were helpful in excluding a diagnosis of AVM.

Lymphangioma can mimic hemangioma microscopically by virtue of similar appearance of small lymphatic channels to capillaries. The lack of erythrocytes in the lumen helps to distinguish between lymphatic channels and capillaries [8]. In the present case, cavernous structures were filled with erythrocytes. Hence, the diagnosis of lymphangioma was excluded.

Vascular leiomyoma (angioleiomyoma) is an uncommon type of leiomyoma very rarely encountered in the uterus. It originates from smooth muscle cells and contains thick-walled vessels [11,12]. Histologically, it is composed of interlacing fascicles of spindle to plump cells swirling around interspersed abundant thick-walled muscular vessels [12]. These features were not consistent with the present case; therefore, angioleiomyoma was not considered as a final diagnosis.

Angiomyofibroma (also called hybrid tumor) is another entity in the differential diagnosis of the cavernous hemangioma of the uterus. In angiomyofibroma, the thick cuffs of onion-skin perithelial smooth muscle cells in the vascular walls admixed with a variably prominent collagen mantle is characteristic and often leads to complete obliteration of the vascular lumens. The abundant deposition of collagen as keloidal bundles is a typical finding in angiomyofibroma [13]. In our case, the lumen of cavernous structures were open and filled with erythrocytes and the intravascular stroma was looser. Therefore, the diagnosis of angiomyofibroma was eliminated.

Isolated uterine hemangiomas may be treated with a variety of options, such as carbon dioxide laser excision, knife excision, cryotherapy, radiotherapy, electrocauterization, internal artery ligation, uterine artery embolization (UAE), local excision, conization, and laser ablation. If hemangiomas are refractory to conservative treatments, hysterectomy may be considered [7]. In the present case, total abdominal hysterectomy was performed due to the excessive vaginal bleeding and existence of an intramural $5.5 \mathrm{~cm}$ mass (myoma uteri).

Pathological examination is necessary for diagnosis of uterine hemangiomas because vaginal examination, ultrasonography, and uterine curettage often remain inadequate to provide clear information about these lesions [8,9]. Immunohistochemical stains such as
CD31 and CD34 can be used to determine the endothelial origin of hemangioma cells [8]. In our case, pathological diagnosis was supported with positive immunohistochemical CD31 staining.

To the best of our knowledge, the current literature contains no reports of cavernous hemangioma arising in an uterine leiomyoma. In this report, we present this unique case with based on its rarity and we suggest that this condition should be kept in mind in differential diagnosis of patients with abnormal vaginal bleeding.

\section{References}

1. Saeed-Vafa D, Myers E, Huang Y, Ferriss JS, Manucha V (2011) Localized cavernous hemangioma of the uterus involving adenomyotic foci. $J$ Cancer Res Ther 7: 69-71. [Crossref]

2. Comstock CH, Monticello ML, Johnson TW, Wechter D (2005) Cavernous hemangioma: diffuse enlarged venous spaces within the myometrium in pregnancy. Obstet Gynecol 106: 1212-1214. [Crossref]

3. Riggs J, Bertoni M, Schiavello H, Weinstein A, Kazimir M (2003) Cavernous hemangioma of the cervix with intractable bleeding. A case report. J Reprod Med 48: 741-743. [Crossref]

4. Bhavsar T, Wurzel J, Duker N (2012) Myometrial cavernous hemangioma with pulmonary thromboembolism in a post-partum woman: a case report and review of the literature. J Med Case Rep 6: 397. [Crossref]

5. Lee EJ, Kim SH, Kim YH (2011) Uterine cavernous haemangioma in a post-menopausal woman: $\mathrm{CT}$ and MRI findings mimicking uterine myoma with degeneration. $\mathrm{Br} J$ Radiol 84: 68-71. [Crossref]

6. Sharma JB, Chanana C, Gupta SD, Kumar S, et al. (2006) Cavernous hemangiomatous polyp: an unusual case of perimenopausal bleeding. Arch Gynecol Obstet 274: 206208. [Crossref]

7. Vijayakumar A, Srinivas A, Chandrashekar BM, Vijayakumar A (2013) Uterine vascular lesions. Rev Obstet Gynecol 6: 69-79. [Crossref]

8. Chou WY, Chang HW (2012) Uterine hemangioma: a rare pathologic entity. Arch Pathol Lab Med 136: 567-571. [Crossref]

9. Malhotra S, Sehgal A, Nijhawan R (1995) Cavernous hemangioma of the uterus. Int $J$ Gynaecol Obstet 51: 159-160. [Crossref]

10. Johnson C, Reid-Nicholson M, Deligdisch L, Grinblat S, Natarajan S (2005) Capillary hemangioma of the endometrium: a case report and review of the literature. Arch Pathol Lab Med 129: 1326-1329. [Crossref]

11. Sahu L, Tempe A, Agrawal A (2012) Angioleiomyoma of uterus. J Obstet Gynaecol 32 713-714. [Crossref]

12. Garg G, Mohanty SK (2014) Uterine angioleiomyoma: a rare variant of uterine leiomyoma. Arch Pathol Lab Med 138: 1115-1118. [Crossref]

13. Jakobiec FA, Zakka FR, Papakostas TD, Fay A (2012) Angiomyofibroma of the orbit a hybrid of vascular leiomyoma and cavernous hemangioma. Ophthal Plast Reconstr Surg 28: 438-445. [Crossref]

Copyright: (C2017 Celik ZE. This is an open-access article distributed under the terms of the Creative Commons Attribution License, which permits unrestricted use, distribution, and reproduction in any medium, provided the original author and source are credited. 